\title{
A REVIEW OF DIFFERENT ALGORITHMS USING IN COLOR IMAGE COMPRESSION
}

\author{
Rashmi Jain ${ }^{1}$, Mahima Jain ${ }^{2}$ \\ PG Scholar ${ }^{1}$, Department of Computer Science \& Engineering, BIST, Bhopal, India \\ Assistant Professor ${ }^{2}$, Department of Computer Science \& Engineering, BIST, Bhopal, India
}

\begin{abstract}
Digital Color Image required substantial measure of space to store and vast data transmission to transmit it. Because of constraints in data transfer capacity and away space, it is basic necessity to packs computerized shading Image. Shading Image compression is required with irrelevant misfortune in Image quality for the compelling utilization of further restored Image. To meet this, various Image compression systems are created in most recent quite a long while. This paper investigations these systems and presents a compression among them to discover the best strategy for computerized shading Image compression. The aim of this paper is to be find out the best approach for growing better approach for advanced color Image compression with least loss in Image quality.
\end{abstract}

Index Terms-Image Compression, Digital Color Image, JPEG, DCT, Wavelet.

\section{INTRODUCTION}

The methodology of high speed enlisting devices and fast change in the field of correspondence has made a gigantic open entryway for various machine based Image applications. The measure of data expected to store an advanced Image is constantly growing and overwhelming the stockpiling devices. Image compression is securing Images using lesser number of bits than its special size. Increase in Image related application has made an issue of Image securing and transmission. Securing and transmission of Images oblige great measure of space and transfer speed. Image compression addresses this issue. It reduces the amount of bits expected to address the Image. In this manner, in the time of computerized correspondence, Image compression is key field of examination [1]. There are two sorts of compression strategies: Lossless compression and Lossy compression. In lossless compression, recreated Image is definitely same as packed Image i.e if data have been losslessly compacted, the principal data can be recovered absolutely from the packed data. It is overall used for applications that can't allow any difference between the first and recreated data [2].

In lossy compression high compression proportion is proficient to the detriment of a couple botches in reproduced Image. It is constantly not vital to get cautious Image after diversion. Image with awesome perceptual quality with some goof is commendable in a couple of uses. For example, it is commendable that a remade highlight sign is not the same as the main the length of the qualifications don't realize chafing ancient pieces. On the other hand, we can generally obtain higher compression proportions than is possible with lossless compression. With stripped eyes, viewer can't perceive the complexity between exceptional Image and decompressed Image. Subsequently generally lossy compression is favored over lossless compression. Most by and large used lossy compression technique is change coding, for instance, Discrete Cosine Transform (DCT) used as a piece of JPEG and
Wavelet change used as a piece of Jpeg2000. Compression proportion and Image nature of decompressed Image, these are two huge things to be considered in Image compression. As compression proportion grows, nature of reproduced Image starts ruining. Various compression techniques like Vector Quantization (VQ), Predictive Coding (PC), Transform Coding (TC), Differential Image Coding (DIC) have been introduced [3]. In change coding, at first DCT was pervasive Image compression technique. DCT indicates straightforwardness and adequate execution in compression. As it is associated on blocked Image relationship over as far as possible can't be murdered. It presents blocking collectibles especially at low piece rate. This drawback was overcome by wavelet change. Since last a few decades wavelets have come into Image and transformed into an engaging methodology for Image compression. It gives time and recurrence examination of data. Wavelet change can be particularly associated with whole Image without blocking it. Wavelet based coding is all the more intense under transmission and interpreting slips. Multiresolution property of wavelet changes see the Image at unmistakable scales. Late example is to use half and half system for Image compression. In crossover strategy, one change is united with a substitute change to fuse the ideal circumstances of both changes. On occasion blend of VQ and change also has been used [4]-[7].

\section{IMAGE COMPRESSION TECHNIQUES AND ALGORITHMS}

Image coder framework contains three associated parts specifically source encoder, quantizer and entropy encoder. Image compression comprises of applying a transform to decorrelate the source information, for example, image or feature, quantizing the transform coefficients lastly changing over the quantized qualities to entropy in Fig. 1. Taking into account processing calculations, compression methods are separated into two noteworthy classifications, for example, 


\section{IJO-SCIENCE}

\section{(INTERNATIONAL JOURNAL ONLINE OF SCIENCE)}

misfortune / lossless based compression and predication / transform based compression.

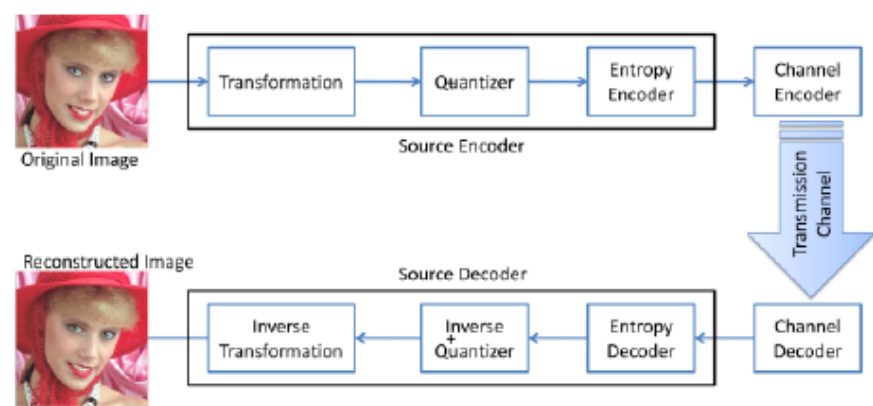

Figure 1: Typical Image Compression and transmission system

\subsection{Lossless Compression}

Lossless compression is numerically indistinguishable to the first image. It is overall called as silent compression. It is characterized into Huffman coding, Arithmetic decomposition, Lempel zin and Run Length Encoding (RLE) .

\subsection{Lossy Compression}

Computations that restore the presentation to resemble the main Image are called lossy frameworks. Amusement of Image is a nearby estimation of one of a kind Image, so the need of estimation of nature of the Image for lossy compression methodology. It gives high compression proportion and as often as possible associated in highlight and Image compression than lossless frameworks. It is described into recurrence orchestrated techniques, desire based techniques and importance arranged methodologies. Information authoritatively sent to foresee coming about qualities by viewing past qualities, for instance, development compensation, Adaptive advanced heartbeat code balance is called farsighted Based techniques. The Image is changed to spatial territory or recurrence space, for instance, Discrete Cosine Transform (DCT), sub band decay, wavelet changes and diverse changes, which gives more unmistakable data compression at the expense of more paramount handling is called Frequency organized systems. The reason for compression uses diverse characteristics of Images, for instance, filtering for case DVI system uses to shading up data isolating and lookup tables, sub analyzing, bit task, quantization, for instance, scalar and vector are called centrality organized methods[9]-[10].

\subsection{Hybrid Compression Techniques}

JPEG, MPEG and Kohonen's Self Organizing Feature Map (SOFM), Set Partitioning in Hierarchical Trees (SPIHT) combine various approaches such as Vector Quantization (VQ) compression and DCT based compression or Differential Pulse Code Modulation (DPCM).

\subsection{Discrete Cosine Transform based Compression} DCT is a Fourier-related change like the discrete Fourier change (DFT), however using simply veritable numbers. Dcts are proportionate to Dfts of by and large twofold the length, taking a shot at honest to goodness data with even symmetry (since the Fourier change of a honest to goodness and even limit is honest to goodness and even), where in a couple of varieties the information and/or yield data are moved impressively an example. There are eight standard DCT varieties, of which four are consistent. The most generally perceived variety of discrete cosine change is the sort II DCT, which is habitually called basically "the Dct",[1]-[2] its opposite, the sort III DCT, is correspondingly frequently called essentially "the converse DCT" or "the IDCT". Two related changes are the discrete sine change (DST), which is equivalent to a DFT of certifiable and odd limits, and the adjusted discrete cosine change (MDCT), which is engaged around a DCT of covering data.

The DCT is used as a piece of JPEG Image compression, MJPEG, MPEG, DV, Daala, and Theora highlight compression. There, the two-dimensional DCT-II of N X N squares are enrolled and the outcomes are quantized and entropy coded. For this circumstance, $\mathrm{N}$ is ordinarily 8 and the DCT-II mathematical statement is associated with every one segment and area of the piece. The outcome is a $8 \times 8$ change coefficient show in which the $(0,0)$ segment (upper left) is the DC (zero-recurrence) part and segments with extending vertical and level record qualities address higher vertical and level spatial frequencies [3].

\subsection{Reversible Integer Discrete Cosine Transforms (RDCT)}

Image compression is accomplished utilizing RDCT. It is gotten from the network factorization hypothesis. Existing Techniques focused around the discrete cosine transform to experience the ill effects of poor execution when contrasted and wavelet image codec in lossy image compression. RDCT joined with different dynamic codec, for example, SPHIT and SPECK. The coefficients are revamped into sub-band structure and coded by setting based piece coding. The DWT based SPHIT supplanted by RDCT with channels. It gives elite in both lossless/lossy compression and utilized for square source pixels good for piece transform by prefilter and utilized for decreasing piece relics by post channel [4].

\subsection{Integer DCT and I2IDCT}

In lossless compression, the rough guess of DCT matrix, the low cost 8-point number DCT (IntDCT) is focused around Walsh Hadamard Transform (WHT) and number lifting. I2idct: The $\mathrm{N}$ point I2I-DCT is accomplished by applying recursive systems and lifting procedures where $\mathrm{N}$ is in force of two for lossless compression and this is proposed by abhayaratne [5]-[7].

\subsection{Fractal Compression}

Fractal compression is a lossy compression system for digital images, in light of fractals. The strategy is best suited for compositions and common images, depending on the way that parts of an image regularly take after different parts of the same image. Fractal calculations change over these parts into scientific information called "fractal codes" which are utilized to reproduce the encoded image. 


\section{IJO-SCIENCE}

\section{(INTERNATIONAL JOURNAL ONLINE OF SCIENCE)}

It is great scientific encoding casing. The premise for this compression is the estimate of unique image focused around arrangement hypothesis and settled point hypothesis for the development of a nearby Iterated Function System (IFS). Diverse strategies have considered for parallel encoding phase of this compression, for example, granularity, information parcel and burden adjusting [8]. Fractal Methodology uses determination. The compression ratio is more prominent than $16 \%$ in 1992 and the drawback is the slow encoding system.

\subsection{EZDCT and TDCT}

Discrete cosine based installed image coder is called Embedded Zero Discrete Cosine Transform (EZDCT) for lossy compression. Tran et al. outline Time Domain Lapped Transform (TDLT) and it is attained by adding preand post channel to the DCT.

\subsection{Discrete Wavelet Transform based Compression}

Much of the time banners we wish to process are in the timezone, however to process every one of them the all the more easily other information, for instance, recurrence is required. Numerical changes decipher the information of signs into particular representations. Case in point, the Fourier change changes over a sign between the time and recurrence spaces, such that the frequencies of a sign can be seen. However the Fourier change can't give information on which frequencies happen at specific times in the sign as time and recurrence are seen uninhibitedly. To deal with this issue the Short Term Fourier Transform (STFT) introduced the considered windows through which unmistakable parts of a sign are seen. For a given window in time the frequencies can be seen. However Heisenburg's Uncertainty Principle communicates that as the determination of the sign upgrades in the time space, by zooming on assorted portions, the recurrence determination crumbles. Possibly, a method for multiresolution is required, which permits certain parts of the sign to be resolved well in time, and diverse parts to be resolved well in recurrence [11]. The power and charm of wavelet examination is accurately this multiresolution. Images contain a great deal of information that obliges much storage space, enormous transmission data transfer capacities and long transmission times. Consequently it is useful to pack the Image by securing only the crucial information anticipated that would duplicate the Image. A Image can be viewed as a framework of pixel (or force) values. Remembering the final objective to brace the Image, redundancies must be mishandled, for locales where there is no change between pixel values. Therefore Images having endless zones of uniform shade will have considerable redundancies, and then again Images that have progressive and tremendous changes in shading will be less abundance and harder to pack [12].

Wavelet examination can be used to detachment the information of a Image into evaluation and unpretentious component and subsignal. Subsignal exhibits the general example of pixel qualities, and three unobtrusive component subsignals show the vertical, even and inclining purposes of hobby or changes in the Image. If these purposes of hobby are minimal then they can be set to zero without by and large changing the Image. The value underneath which inconspicuous components are seen as adequately little to be set to zero is known as the edge. The more noticeable the amount of zeros the more essential the compression that can be achieved. The measure of information held by a Image after compression and decompression is known as the imperativeness held and this is relating to the aggregate of the squares of the pixel values. In case the imperativeness held is $100 \%$ then the compression is known as lossless, as the Image can be remade exactly. This happens when the edge worth is arranged to zero, suggesting that the inconspicuous component has not been changed. If any qualities are changed then imperativeness will be lost and this is known as lossy compression. Possibly, in the midst of compression the amount of zeros and the essentialness support will be as high as could be normal the situation being what it is. In any case, as more zeros are gotten more essentialness is lost, so a concordance between the two should be found

\subsection{VQ Compression}

Vector Quantization is the most ideal method for quantizing and layering the images. The central thought is to secure a codebook comprising of code vectors such that each one code vector can speak to a set of image pieces of size $m \times m$. An image is first segment into $\mathrm{m} \times \mathrm{m}$ non-covering square, which is spoken to as $\mathrm{m} 2$-tuple vectors are called preparing vectors. Vector Quantization (VQ) gives preferable execution over Scalar Quantization (SQ). SAR image work better for VQ compression since quantization is first approach for SAR images. The essential work is to plan an adaptable Codebook and Nasrabadi and King gives a decent survey of VQ. Chen's correlation shows that a codebook created focused around (Linde, Buzo and Gray) LBG calculation has higher PSNR values over some different plans slow disconnected from the net preparing [14]. It is the straightforward decoder and preferences are no coefficient quantization and not proper for low bit rate compression, the measure of reckonings amid the quest for ideal code vector in encoding little bits every pixel furthermore slow codebook generation or slow disconnected from the net preparing. The compression ratio is short of what $32 \%$.

\subsection{Hybrid Wavelet Transforms}

The idea of producing hybrid wavelet transform from two orthogonal transforms and amplifies it with diverse orthogonal transform like DCT, WALSH, DHT, DKT and so forth. Creating wavelet transform from orthogonal transform frameworks of two separate sizes from two orthogonal transform networks AM of size MxM as indicated in Fig.2 and $\mathrm{BN}$ of size $\mathrm{NxN}$ as demonstrated in Fig.3, we can generate wavelet transform matrix $\mathrm{T}$ of size $\mathrm{MNxMN}$. Case in point, from orthogonal transform matrix AM of size $32 \times 32$ and $B$ of size $8 \times 8$, we can generate wavelet transform matrix of size $256 \times 256$. To generate first $\mathrm{N}$ lines of wavelet transform matrix, duplicate every segment of matrix $\mathrm{B}$ with every 


\section{IJO-SCIENCE}

\section{(INTERNATIONAL JOURNAL ONLINE OF SCIENCE)}

coefficient of the first line of matrix A. To generate next $\mathrm{N}$ lines, second column of AM is affixed with zeros and afterward it is movement pivoted [15].

\section{LITERATURE REVIEW}

Image compression utilizing wavelet change is engaged by numerous specialists since most recent two decades.

M. S. Abdullah and N. Subba Rao [29] have proposed examination of various wavelets for Image compression. Daubechies wavelet [30], Haar wavelet, Coiflet, Symlet and bi orthogonal Wavelet changes have been utilized to think about their exhibitions.

Wei Pin Lin et al. proposed a technique [31] to anticipate the information of high recurrence from an annihilated Image with indicated down inspecting principle. It abuses the relationship between's low pass and high pass yields which can be utilized to pack the Image and get great quality recreated Image.

Tree based wavelet Image coder is proposed by Jose Oliver, Manuel P. Malumbres [32]. In this technique wavelet tree is shaped by sensibly orchestrating change coefficients in order to uproot the repetition among the sub groups.

Arian Maleki et al. have proposed Image compression utilizing directional wavelet changes [33]. To dodge the disadvantage of quad tree parceling, they have utilized new plan of apportioning called uber quad dividing.

Shih-Chung B. Lo, Huai Li, and Matthew T. Freedman have proposed neural system based wavelet change for Image compression [34]. They have utilized straight convolution neural system to look for the wavelet that minimizes the blunder and expands the effectiveness. Daubechies wavelet and Haar wavelet have been utilized by them for compression. A half and half Image compression method actualizing a fourdimensional change consolidating the discrete wavelet change and the discrete cosine change has been proposed by $\mathrm{E}$. Elharar et al. [35]. It is utilized for basic imaging and beats JPEG compression plan connected to indispensable Images. Image compression utilizing double tree complex wavelet change is displayed by Li Hui Fang et al. [36]. It utilizes SPIHT calculations, which depends on not just the relationship between the bit-planes and the objective piece rate additionally the relationship between the underlying edge and the objective piece rate.

Blend of fractal Image encoding and wavelet decay is proposed in by Xiao-Yan $\mathrm{Xu}$ et al. [37]. In their proposed procedure of encoding, the Image is managed by three level wavelet deterioration. High recurrence area of the deteriorated Image embraces scathless anticipating encoding, while the low recurrence segment receives fractal compression encoding. During the time spent unraveling, the high recurrence segment completes scorch less anticipating translating, while the low recurrence segment is decoded to iterative capacity framework code which is utilized for subimage recreation.

Compression utilizing cross breed change is displayed as a part of [38]. Kronecker result of Kekre change with other orthogonal changes is utilized and blunder at various compression proportions is watched. It has been watched that when DCT is joined with Kekre change gives least blunder as DCT has high vitality compaction property than other orthogonal changes.

Basic wavelet era strategy proposed by Kekre et al. [39] is utilized to contrast execution of wavelet changes with separate orthogonal changes [40]. It has been watched that wavelet changes of orthogonal changes give preferable compression over individual orthogonal change. Next headway to wavelet changes is mixture wavelet change that utilizations two distinctive orthogonal changes to create new change framework. As of late half breed wavelet changes have been contemplated and ended up being superior to anything wavelet change in numerous Image handling applications including Image compression.

Compression utilizing Real Fourier-DCT half breed wavelet change is proposed by Kekre et al. [41]. It demonstrates that when RealDFT and DCT are consolidated to create mixture wavelet, it gives preferable execution over compression got by individual DCT or Real DFT.

It is additionally superior to anything DCT wavelet change [33] .Hybrid wavelet of Haar and non sinusoidal changes has been proposed in [42]. Distinctive sizes of part changes have been attempted and one giving better Image quality has been chosen.

Then again Haar change matched with sinusoidal changes is utilized for Image compression [43]. Haar change matched with DCT gives predominant execution than its blend with DST, Hartley and Real-DFT.

\section{CONCLUSION}

The study outlines the requirement for compression and arrangement of compression strategies. A review is performed on the most fundamental and development compression strategies, including coding procedures in light of DCT, DWT, VQ, Fractal approach and different techniques. The compression between Wavelet, JPEG, VQ and Fractal methodology is additionally done in writing. Every one of these studies demonstrate that the wavelet approach appears to be vastly improved than different methodologies. The mixture wavelet approach by utilizing DCT, DKT and DHT is another best approach for productive lossles compression.

\section{REFERENCES}

[1] Sahav Singh Yadav and Sanjay k. Sharma, "Efficient Image Compression using all the Coefficients of 16x16 DCT Image Sub-Block", International Journal of Engineering and Innovative Technology (IJEIT) Volume 4, Issue 8, February 2015

[2] P.Lohitha And T.Ramashri, "Color Image Compression Using Hierarchical Prediction Of Pixels", International Journal Of Advanced Computing And Electronics Technology(Ijacet), Issn(Print):2394-3408,(Online):2394-3416,Volume-2,Issue1,2015 


\section{IJO-SCIENCE}

\section{(INTERNATIONAL JOURNAL ONLINE OF SCIENCE)}

[3] A.H.M. Jaffar Iqbal Barbhuiya, Tahera Akhtar Laskar and K. Hemachandran, "AN APPROACH FOR COLOR IMAGE COMPRESSION OF BMP AND TIFF IMAGES USING DCT AND DWT", INTERNATIONAL JOURNAL OF COMPUTER ENGINEERING \& TECHNOLOGY (IJCET), Volume 6, Issue 1, January (2015), pp. 19-26

[4] Priyatosh Halder and Suman, "A LOSSLESS COLOR IMAGE COMPRESSION USING AN IMPROVED REVERSIBLE COLOR TRANSFORM WITH HIERARCHICAL PREDICTION AND MODIFIED HUFFMAN CODING", International Journal of Research in Engineering and Technology eISSN: 2319-1163 | pISSN: 2321-7308,

[5] D. Venugopal, M.Gunasekaran, A.Sivanatharaja, "Secured Color Image Compression and Efficient Reconstruction Using Arnold Transform with Chaos Encoding Technique", International Journal of Advanced Research in Computer and Communication Engineering, Vol. 4, Issue 5, May 2015

[6] Chunxiao Zhang, Chengyou Wang and Baochen Jiang, "Color Image Compression Based on Directional All Phase Biorthogonal Transform”, International Journal of Multimedia and Ubiquitous Engineering Vol. 10, No. 1 (2015), pp. 247-254

[7] Gaganpreet Kaur and Priyanka Jarial, "A Survey On DCT And Fuzzy Image Compression Algorithms", Imperial Journal of Interdisciplinary Research (IJIR) Vol-2, Issue-6, 2016 ISSN: 2454-1362

[8] Manoj Kumar and Ankita Vaish, "Lossy Compression of Color Images using Lifting Scheme and Prediction Errors", I.J. Modern Education and Computer Science, 2016, 4, 1-8 Published Online April 2016

[9] Manjit Sandhu, Jaipreet Kaur, Sukhdeep Kaur, "Matlab Based Image Compression Using Various Algorithms", International Journal of Advanced Research in Computer Science and Software Engineering, Volume 6, Issue 4, April 2016

[10] Poonam Dhumal and S. S. Deshmukh, " Survey on Comparative Analysis of Various Image Compression Algorithms with Singular Value Decomposition", International Journal of Computer Applications (0975 - 8887) Volume 133 - No.6, January 2016

[11] Kumar, S., and Sood, V., Quality Assessment of colour image compression using Haar Wavelet Transform, International Journal of Engineering Trends and Technology, 266-269, 2012.

[12] Kekre, H.B., Sarode, T., and Natu, P., Image Compression Using Real Fourier Transform, Its wavelet Transform and Hybrid Wavelet Transform, International Journal of Advanced Computer science and Application, 2013.

[13] Sunil, Kumar, V.V., and Reddy, M.I.S, Image compression technique by using wavelet transform, journal of Information Engineering and applications, 2012.

[14] Nadenau, M.J., Reichel, J., and Kunt, M., Wavelet Based Colour Image Compression: Exploiting the Contrast Sensitivity Function, IEEE Transactions Image Processing.
[15]Hekre, H.B., Sudeep, D., Thepade, and Parkar, A., A Comparison of Haar Wavelets and Kekree"s Wavelets for Storing Colour Informationin a Greyscale Image, International Journal of Computer Applications (IJCA),32-38, 2010.

[16] Hekre, H.B., Sudeep, D., Thepade, and Maloo, A., Performance Comparison of Image Retrieval Techniques using Wavelet Pyramids ofWalsh, Haar and Kekre Transforms, International Journal of Computer Applications (IJCA), 1-8, 2010.

[17] Hekre, H.B., Sudeep, D., Thepade, and Maloo, A., Face Recognition using Texture Features Extracted form Walshlet Pyramid, ACEEE International Journal on Recent Trends in Engineering and Technology (IJRTET), 2010.

[18] Hekre, H.B., Sudeep, D., Thepade, and Maloo, A., Face Recognition using Texture Features Extracted from Haarlet Pyramid, International Journal of Computer Applications (IJCA), 41-45, 2010.

[19] Hekre, H.B., Sudeep, D., Thepade, and Natu, P., Image Compression based on Hybrid wavelet transform generated using orthogonal component transform of different sizes, International Journal of soft computing and Engineering, 2013.

[20] Walsh, J.L., A Closed Set of Orthogonal Functions, AmericanJournal of Mathematics, 5-24, 1923.

[21] Ahmed, N., Natarajan, T., and Rao, K. R., Discrete CosineTransform, IEEE Transaction Computers, 90-93, 1974.

[22] Chen, W., Smith, C. H., and Fralick, S. C., A Fast Computational Algorithm For The Discrete Cosine Transform, IEEE Transaction Communications, 1004-1008, 1977.

[23] Kekre, H.B., and Thepade, S.D., Image Retrieval using NonInvolutional Orthogonal Kekre's Transform, International Journal of Multidisciplinary Research and Advances in Engineering (IJMRAE), 189-203, 2009.

[24] Kekre, H.B., and Thepade, S.D., Athawale, A., Anant S., Prathamesh V., and Suraj S., Kekre Transform over Row Mean, Column Mean and Both using Image Tiling for Image Retrieval, International Journal of Computer and Electrical Engineering (IJCEE), 964-971, 2010.

[25] Hartley, R. V. L., A more symmetrical Fourier analysis applied to transmission problems, Proceedings of IRE 30, 144-150, 1942.

[26] Strang G. "Wavelet Transforms Versus Fourier Transforms." Bull. Amer. Math. Soc. 28, 1993, 288-305.

[27] N. Ahmed, T. Natarajan and K. R. Rao, "Discrete Cosine Transform”, IEEE Transaction Computers, January 1974, C-23, pp. 90-93.

[28] S. Mallat, "A Theory of Multiresolution Signal Decomposition: The Wavelet Representation," IEEE Trans. Pattern Analysis and Machine Intelligence, vol. 11, pp. 674-693, 1989.

[29] M.S. Abdullah, N. Subba Rao, "Image Compression using Classical and Lifting based Wavelets", International Journal of Advanced Research in Computer and Communication Engineering, August 2013, Vol. 2, Issue 8, pp. 3193-3198. 


\section{IJO-SCIENCE}

\section{(INTERNATIONAL JOURNAL ONLINE OF SCIENCE)}

[30] Daubechies, I. "The wavelet transform, time-frequency localization and Signal analysis", IEEE Transformation and Information Theory 36: 1990, 961-1005.

[31] Wei-Pin Lin, Chih-Ming Chen, and Yung-Chang Chen, "Image Compression with Interpolation in Wavelet Transform Domain" 2005. IEEE International Symposium on Circuits and Systems (ISCAS), 2005, Vol. 3, pp. $2084-2087$.

[32] Jose Oliver, Manuel P. Malumbres, "Low Complexity MultiResolution Image Compression using Wavelet Lower Trees", IEEE Trans. on Circuits and Systems for Video Technology, Nov. 2006, Vol. 16, Issue 11, pp. 1437-1444.

[33] Maleki A, Rajaei B, Pourreza H.R., "Rate Distortion Analysis of Directional Wavelets", IEEE Trans. on Image Processing, Feb 2012, Vol. 21 Issue 2, pp. 588-600.

[34] Shih-Chung B. Lo, Huai Li, and Matthew T. Freedman, "Optimization of Wavelet Decomposition for Image Compression and Feature Preservation", IEEE Trans. on Medical Imaging, Vol. 22, Issue 9, Sept 2013, pp. 1141-1151.

[35] E. Elharar, Adrian Stern, Ofer Hadar, and Bahram Javidi, “A Hybrid Compression Method for Integral Images using Discrete Wavelet Transform and Discrete Cosine Transform", Journal Of Display Technology, September 2007, Vol. 3, No. 3, pp. 321325.

[36] Jingyu Yang, Wenli Xu, Qionghai Dai, Yao Wang, "Image Compression using 2D Dual Tree Discrete Wavelet Transform (DDWT)", IEEE International Symposium on Circuits and Systems, May 2007, pp. 297-300.

[37] Xiao-Yan Xu, Chen, P., Juan Dai, "Hybrid Encoding Analysis of Fractal Image Compression Method Based on Wavelet Transform", In Proc of International Conference of Machine Learning and Cybernetics, 2008, Vol. 5, pp. 2886-2889.

[38] H.B. Kekre, Tanuja Sarode, Prachi Natu, "Digital Image Compression using Hybrid Transform with Kekre Transform and Other Orthogonal Transforms", Journal of Computer Engineering, Jan. 2014, Vol. 16, Issue 1, Ver. III, PP 38-46.

[39] H. B. Kekre, Tanuja Sarode, Sudeep Thepade, Sonal Shroff, "Instigation of Orthogonal Wavelet Transforms using Walsh, Cosine, Hartley, Kekre Transforms and their use in Image Compression", International Journal of Computer Science and Information Security, 2011, Vol. 9, No. 6, pp. 125-133.

[40] H.B. Kekre, Tanuja Sarode, Prachi Natu, “ Image Compression Using Column, Row and Full Wavelet Transforms Of Walsh, Cosine, Haar, Kekre, Slant and Sine and Their Comparison with Corresponding Orthogonal Transforms". International Journal of Engineering Research and Development (IJERD), Mar.2013, Vol. 6. Issue 4, pp. 102-113.

[41] H.B. Kekre, Tanuja Sarode, Prachi Natu, "Image Compression using Real Fourier Transform, It's Wavelet Transform and Hybrid Wavelet with DCT", International Journal of Advanced Computer Science and Applications (IJACSA), 2013, Vol. 4, No.5, pp. 41-47.
[42]H.B. Kekre, Tanuja Sarode, Prachi Natu, "Color Image Compression using Hybrid Wavelet Transform with Haar as Base Transform", International Journal of Scientific and Research Publications, June 2014, Volume 4, Issue 6, pp. 1-13.

[43]H.B. Kekre, Tanuja Sarode, Prachi Natu, "Performance Comparison of Hybrid Haar Wavelet Transform with Various Local Transforms in Image Compression using Different Error Metrics", International Journal of Image Processing (IJIP), July 2014, Vol.8, Issue 4, pp. 186-203

[44] A.M.Raid, W.M.Khedr, M. A. El-dosuky and Wesam Ahmed, "HYBRID JPEG COMPRESSION USING COLOR BASED SEGMENTATION", Advances in Vision Computing: An International Journal (AVC) Vol.2, No.3,September 2015 